\title{
PI3K Inhibition Sensitize the Cisplatin-resistant Human Ovarian Cancer Cell OVCAR3 by Induction of Oxidative Stress
}

\author{
Sahar Baghal-Sadriforoush ${ }^{1}$, Morteza Bagheri*2 $^{2}$ Isa Abdi Rad ${ }^{2}$, \\ Fattah Sotoodeh Nejadnematalahi ${ }^{1}$
}

\begin{abstract}
Background: This study evaluates the effect of simultaneous AKT inhibition and cisplatin therapy in changes of Reactive Oxygen Species (ROS) production, apoptosis induction, and cell survival in cisplatinresistant OVCAR3 cell.

Methods: OVCAR3 cancer cells were treated with cisplatin, Ly 294002 (LY), and cisplatin+Ly to investigate the cytotoxicity effect of the mentioned groups via MTT assay. Then, DCFH-DA (2', 7'dichlorodihydro fluorescein diacetate) assay kit is used to assess the potential of treated groups in intracellular ROS generation. Protein expression levels of caspase-3, cleaved caspase 3, PI3K, Akt, p-Akt, XIAP, and Survivin are estimated through immunoblotting assay in all three experimental groups.

Results: The results showed that all three treated groups, including cisplatin and Ly alone and coadministration of cisplatin+Ly, could reduce the cell vitality of OVCAR3 cancer cells, induced intracellular production of ROS and increased the expression level of activated caspase 3 and Akt protein, whereas downregulated the phosphorylation of Akt protein. However, the effect of combination therapy was more tangible compared to single therapy and control groups. In contrast, the expression amount of XIAP, Survivin, and PI3K did not show detectable changes in comparison with the control group.

Conclusions: The results showed that the AKT inhibition by Ly could sensitize the OVCAR3 cancer cells to the cisplatin and lower the effective dose of cisplatin through hyperactivation of oxidative stress.
\end{abstract}

Keywords: Caspase-3, Cisplatin, Ovarian cancer, PI3K/Akt signaling.

\section{Introduction}

Ovarian cancer with the fourth most common cause of cancer-related death can be considered as an aggressive and under-recognized gynecological malignancy in women across the world (1-3). Lack of effective screening methods and little or no specific symptoms resulted in ovarian cancer diagnosis only at an advanced stage (stage III or IV) and, subsequently, a high mortality rate $(2,4)$. Surgical debulking followed by the combination of platinum-based and taxanebased drugs (cisplatin and carboplatin) are first-line treatments used to treat advanced ovarian cancer in clinical practice $(5,6)$. However, the development of chemoresistance and tumor relapse is the primary clinical obstacle that poses a significant impediment, and restriction in optimal cancer therapy led to a considerable reduction in survival rates (7-9). Therefore, a high concentration of platinumbased treatments and secondary chemotherapy drugs are needed to overcome drug resistance resulted in adverse side effects and severe toxicity such as gastrointestinal disorder, 
asthenia, renal, and neurological consequences $(1,10)$. The resistance of ovarian cancer cells to chemotherapy drugs is created firstly due to the complex interaction of multiple factors and multi-gene. For instance, the deregulated apoptosis pathway and also PI3K/Akt pathway are major contributors factors to drug resistance and cancer development (11).

For this reason, it is currently essential to identify a novel approach to improve the efficiency of therapeutic agents and curb their side effects and also molecules to target multiple drug-resistant mechanisms and consequently tackle chemotherapy resistance and increase the sensitivity of cancer cells to chemotherapeutic agents $(12,13)$. Cisplatin is one of the most effective chemotherapy drugs, which is implied in the form of either monotherapy or combination to treat ovarian cancer, induce apoptosis via cysteine proteases called caspases cascades in ovarian cancer cells $(10,11)$. In ovarian cancer, activation of phosphatidylinositol 3kinase/protein kinase B (PI3K/Akt) pathway as a result of down-regulation of phosphatase and tensin homolog (PTEN), RET/PTC oncogenes and activated $\mathrm{p} 21$ plays a vital role in biological processes of ovarian tumor cells including tumorigenesis, proliferation, growth regulation, loss of apoptosis, metabolism as well as resistance to apoptosis (14-17). Strategies aimed at the downregulation of this signaling pathway in combination with conventional anti-cancer drugs might bring about suppression of cell proliferation and cell death and take into account as a suitable approach to overcome chemoresistance in clinical. Hence, LY294002 as an inhibitor of PI3K class I catalytic subunit cause inactivation of this protein led to apoptosis induction and inhibition of cancer cell growth in vitro and in vivo $(2,4)$.

In this study, we aimed to investigate the effects of Ly and cisplatin either alone or simultaneously on ROS generation, apoptosis-related proteins, caspase 3 and xXIAP activation, and cell survival-related proteins $\mathrm{PI} 3 \mathrm{~K}, \mathrm{AKT}$ and surviving regulation.

\section{Materials and Methods Materials}

MTT [3-(4, 5-dimethylthiazol-2-yl)-2, 5diphenyltetrazolium bromide] powder and cisplatin as a conventional anti-cancer drug were purchased from Sigma Chemical Co. (St. Louis, MO). Human ovarian cancer cells line (OVCAR3 cell line NCBI code: C209), were obtained from the National Cell Bank of Iran (NCBI), Pasteur Institute. All cell culture reagents were provided from an appropriate, commercially available supplier. All antibodies were from Santa Cruz Biotechnology Company.

\section{Cell culture}

Cisplatin-resistant ovarian cancer cell line OVCAR3 were incubated at $37{ }^{\circ} \mathrm{C}$ in a humidified atmosphere with 5\% CO2. RPMI 1640 as a standard media (Sigma-Aldrich, USA) containing $10 \%$ heat-inactivated fetal bovine serum (FBS) (Gibco, USA), $100 \mathrm{U} / \mathrm{ml}$ penicillin and $100 \mu \mathrm{g} / \mathrm{ml}$ streptomycin (Gibco, USA) were used routinely to culture cells.

\section{Cell viability assay}

Cancer growth inhibitory effect was demonstrated using (3-(4, 5-Dimethylthiazol2-yl)-2, 5-Diphenyltetrazolium Bromide (MTT) (Sigma-Aldrich, USA) assay. Briefly, $1 \times 10^{4}$ cells/well were seeded into a 96-well plate cultured in RPMI-1640 medium supplemented with $10 \%$ FBS and allowed to grow overnight. Later on, the cells were treated with different concentrations of either cisplatin or Ly in the course of 24,48 , and 72 $\mathrm{h}$ cultured in the same media complemented with $2 \%$ FBS. Next, $20 \mu$ of MTT solution (5 $\mathrm{mg} / \mathrm{ml}$ ) was added to each well and incubated in the dark for another $4 \mathrm{~h}$ at $37^{\circ} \mathrm{C}$. After the aspiration of culture media, $200 \mu 1$ of dimethyl sulfoxide (DMSO) used to solubilize MTT formazan crystals. Optical density (OD) was determined at a wavelength of $630 \mathrm{~nm}$ via a microplate reader (Biotek Instruments, USA). The concentration of cisplatin or Ly caused $50 \%$ inhibition of OVCAR3 cell activity named IC50, which is expressed as (OD 
control-OD experimental)/OD control $\times$ $100 \%$ and calculated via dose-response curve of cell viability percentage against various concentrations of cisplatin or Ly. Regarding either additive or synergistic effect of simultaneous administration of cisplatin and Ly, the IC50 value of Ly after $24 \mathrm{~h}$ combined with various concentrations of cisplatin for 24 $\mathrm{h}$ to determine the impact of this combination on IC50 of Cisplatin. Each evaluation was displayed in triplicate.

\section{Measurement of ROS}

The intracellular ROS production was carried out using a DCFH-DA (2', 7'-dichlorodihydro fluorescein diacetate) assay kit. DCFH is oxidized to a highly fluorescent agent such as dichlorofluorescein (DCF) in the existence of cellular oxidizing agents. OVCAR3 cells were seeded in a 96-well plate and then incubated at $37{ }^{\circ} \mathrm{C}$ in $5 \% \mathrm{CO} 2$ and $95 \%$ air. At the end of incubation time, treated cells were harvested and washed with PBS PH 7.4 for two times Followed by resuspending cells in $100 \mu \mathrm{l}$ of DCFH-D dissolved in a fresh media in the dark for $30 \mathrm{~min}$ at $37{ }^{\circ} \mathrm{C}$ and in a humidified atmosphere containing $5 \% \mathrm{CO} 2$ and allowed activation of dye. After removing the free dye by washing with PBS, the cells were subjected to photography by a fluorescence microscope (Olympus IX70, Tokyo, Japan). The results of ROS production are expressed as the Mean Fluorescence Intensity (RIF) of DCF at excitation and emission wavelengths of 530 $\mathrm{nm}$ and $485 \mathrm{~nm}$, respectively.

\section{Protein extraction and Western blot analysis} Immunoblotting assay (RIPA) used for protein expression analyses. Firstly, treated cells were suspended in $100 \mu 1$ RIPA ice-cold lysis buffer (25 mm HEPES, 1\% Triton X-100, $2 \mathrm{~mm}$ EDTA, $0.1 \mathrm{~m} \mathrm{NaCl}, 25 \mathrm{~mm} \mathrm{NaF}, 1 \mathrm{~mm}$ Sodium Orthovanadate) compromised protease inhibitors during $30 \mathrm{~min}$. Afterward, cell lysates were centrifuged at $12000 \mathrm{~g}$ for 20 min at $4{ }^{\circ} \mathrm{C}$, and the supernatant was collected to obtain total proteins. Equal amounts of each protein sample $(20 \mu \mathrm{g})$, which is quantified via Bradford protein assay were separated with
$12 \%$ Sodium dodecyl sulfate-polyacrylamide gel electrophoresis (SDS-PAGE). They subsequently shifted to polyvinylidene difluoride (PVDF) membrane (Roche, UK). After blocking the membrane at room temperature for $1 \mathrm{~h}$ with bovine serum albumin (BSA) $3 \%$ in Tris-buffered saline ( $\mathrm{pH} 7.5)$ to remove Non-specific binding reactions, the membrane was maintained with diluted primary antibodies, X-linked inhibitor of apoptosis protein (sc-55550), phospho-Akt (sc-52940), Akt (sc-5298), $\beta$-actin (sc-47778), Survivin (sc-17779) and caspase 3 (sc-136219) at $4{ }^{\circ} \mathrm{C}$ overnight. After that, the membrane was washed with PBS and then probed with HRP-conjugated conjugated secondary antibody for $2 \mathrm{~h}$ at room temperature. Eventually, Protein expression was visualized through the enhanced chemiluminescence (ECL) detection kit (Pierce, Rockford, IL). The protein band intensities were pictured and analyzed by Image J software (version 1.62, National Institutes of Health, Bethesda, MD, USA) and normalized to $\beta$-actin as an internal loading control (18).

\section{Statistical analysis}

All the experiments were carried out in triplicate and repeated three times. Statistical analysis was performed using the GraphPad Prism 6 Scientific software (GraphPad Software, Inc., La Jolla, CA). The results were presented as the mean \pm SEM. One-way analysis of variance (ANOVA) followed by Tukey post-hoc test to determine the differences between the experimental groups. A $p$ value of $<0.05$ was considered to indicate statistically significant.

\section{Results MTT assay}

The effect of Ly, cisplatin, and the combination of cisplatin and Ly on cell viability was examined via MTT assay on OVCAR3 cancer cells, and the results showed in Figures 1A, 1B, and 1C. Based on obtained results IC50 value of cisplatin alone became $13.18 \mu \mathrm{M}, 11.55 \mu \mathrm{M}$, and $9.02 \mu \mathrm{M}$ for 24,48 , and $72 \mathrm{~h}$, respectively. Besides, Cis+Ly 
reduced cell viability more than single therapy with an IC50 amount of $5.46 \mu \mathrm{M}, 4.60 \mu \mathrm{M}$, and $4.82 \mu \mathrm{M}$ during 24,48 , and $72 \mathrm{~h}$, respectively, which proved the increased sensitivity of OVCAR3 cells to cisplatin in the presence of $5 \mathrm{uM}$ of Ly (Table 1).

Table 1. Comparison of IC50 amount of cispaltin, cisplatin in combination with Ly. $* \mathrm{p}<0.05$, ** $\mathrm{p}<0.01$ and $* * * \mathrm{p}<$ 0.001 versus control.

\begin{tabular}{cccc}
\hline Time & Cisplatin IC50 & Cisplatin+Ly IC50 & p-value \\
\hline 24 & 13.18 & 5.46 & $\mathrm{p}<0.001$ \\
48 & 11.55 & 4.60 & $\mathrm{p}<0.001$ \\
72 & 9.02 & 4.82 & $\mathrm{p}<0.01$ \\
\hline
\end{tabular}
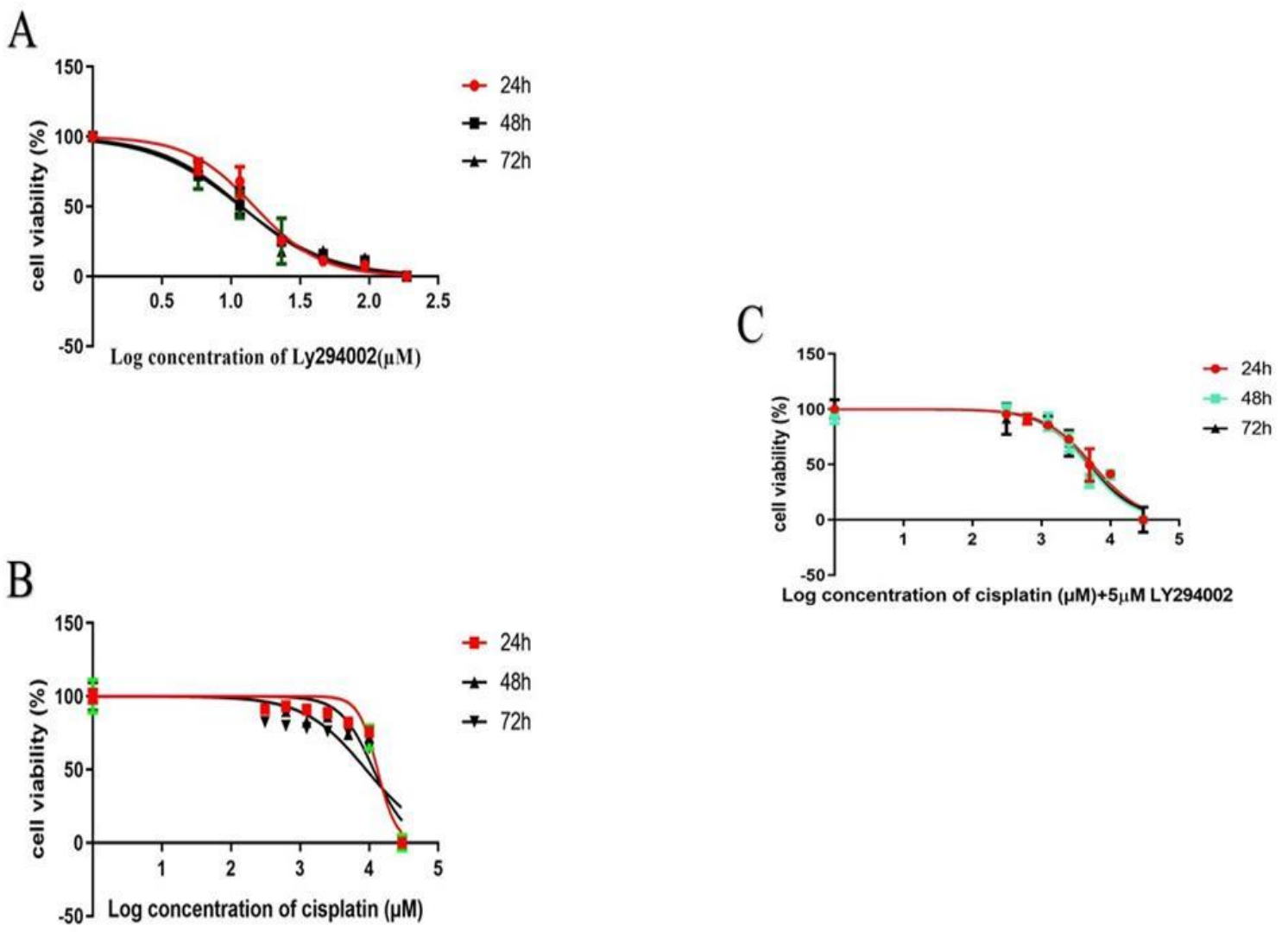

Fig. 1. effect of Ly, Cisplatin, and Cisplatin+Ly on the proliferation of ovarian cancer cells alone or in combination. OVCAR3 cells were treated with a fixed concentration of $5 \mu \mathrm{M}$ of Ly and different concentrations of cisplatin and cisplatin+ Ly during 24 , 48 , and $72 \mathrm{~h}$. Obtained triplicate data were expressed as mean \pm SEM.

\section{Intracellular ROS levels}

H2DCF-DA was used to examine ROS generation in OVCAR3 cells. The results in Fig. 2 revealed that Ly, cisplatin, and coadministration of cisplatin and Ly significantly induced intracellular ROS generation versus control. Among them, combined treatment with cisplatin and Ly displayed considerable augmentation in the ROS level versus to each of them alone $(\mathrm{p}<0.01)$. Hence, the combination of cisplatin with Ly is the better choice for ROS production in OVCAR3 ovarian cancer cells. 
A

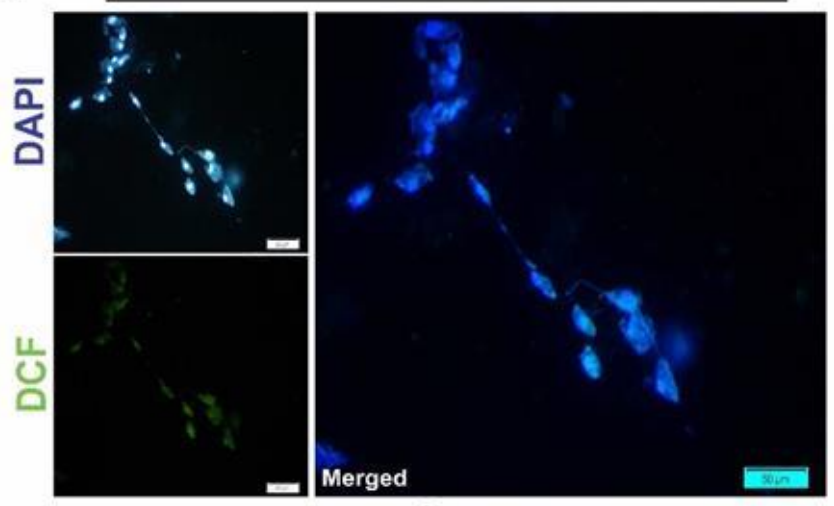

Ly

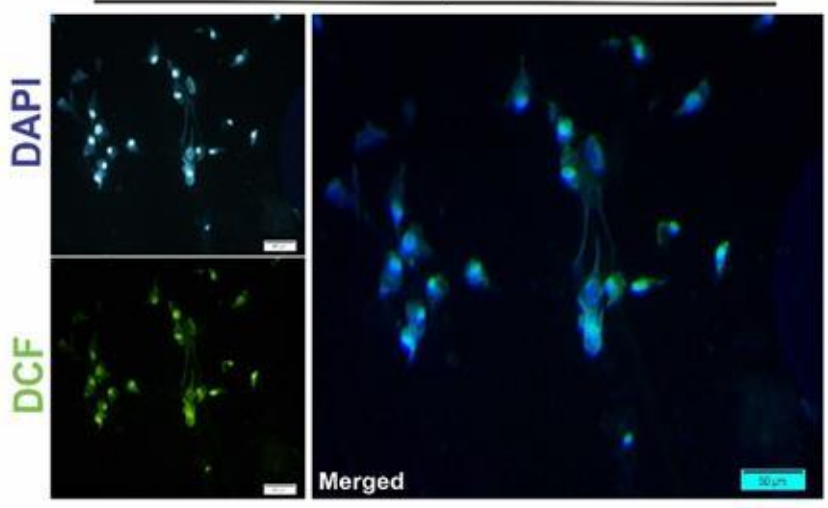

Cis

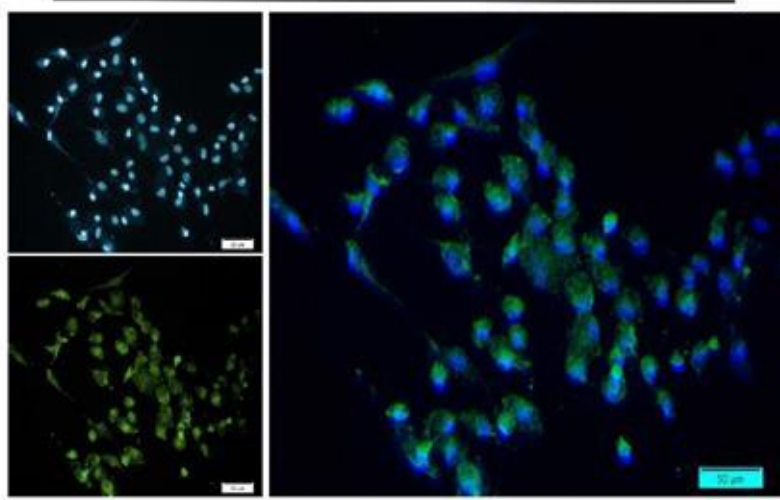

Cis+Ly

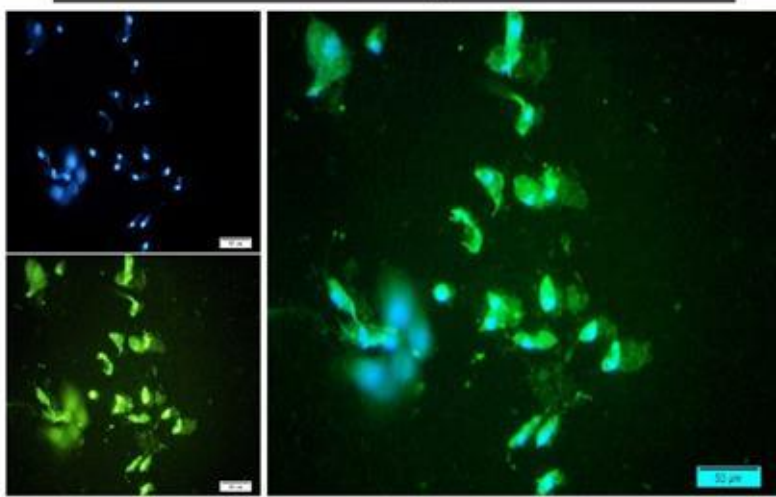

B

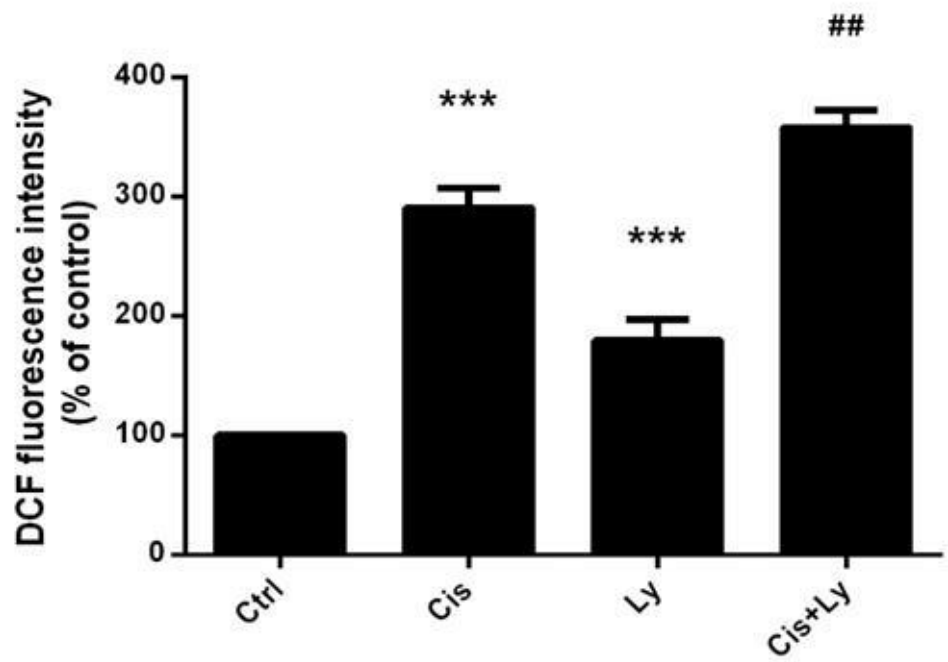

Fig. 2. Intracellular detection of reactive oxygen species (ROS) generation after treatment with cisplatin, Ly, and cisplatin+ LY in OVCAR3 cells. Results are presented as a percentage of the control and express the mean \pm SEM of three independent experiments. ${ }^{*} \mathrm{p}<0.05,{ }^{* *} \mathrm{p}<0.01$ and $* * * \mathrm{p}<0.001$ versus control. \#\# $\mathrm{p}<0.01$ versus cis (Ctrl: control; Cis: cisplatin, Ly, Cis+Ly: Cisplatin+Ly).

\section{Apoptosis markers}

To determine the apoptosis induction in experimental conditions, the expression of apoptosis-related genes was analyzed in treated cells. Based on the obtained results in the western blotting test, treatment with all three 
mentioned groups had no detectable effect on XIAP expression, whereas treatment with cisplatin and Ly alleviated caspase 3 activations ( $\mathrm{p}<0.001)$. However, cisplatin plus Ly showed a considerable decrease in total caspase 3 expressions (cleaved+procaspase3) versus control chemoresistant cells (Fig. 3A). When it comes to changes in activation of caspase 3 , treatment with cisplatin+Ly increased the cleaved caspase 3

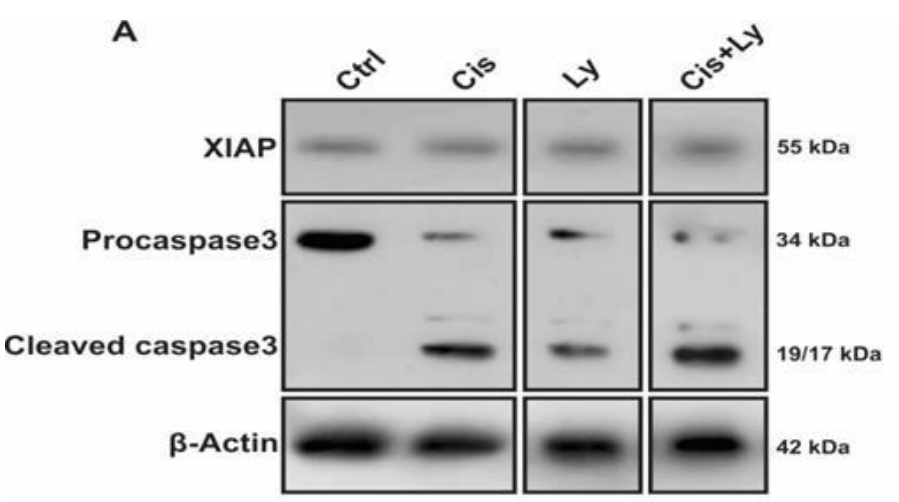

C

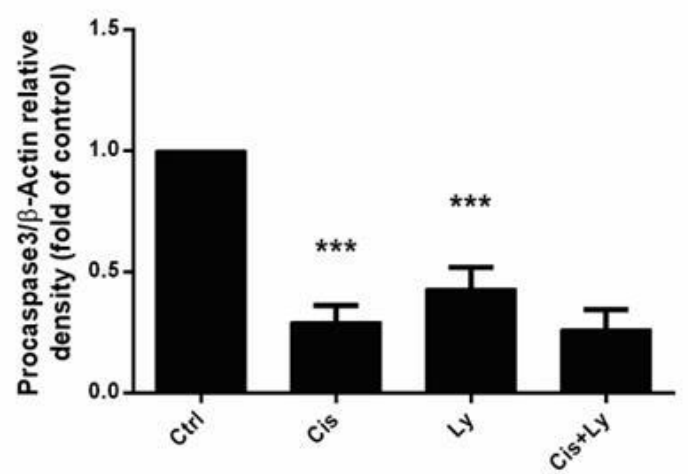

versus Ly $(p<0.05)$; however, no significant change was detected versus cisplatin $(\mathrm{p}=128)$. It seems that the up-regulation of cleavage of caspase 3 protein did not have a remarkable difference in the cisplatin + Ly as compared with cisplatin alone (Fig. 3D). Nevertheless, it is noticeable that approximately half of the concentration of cisplatin has been used in combination conditions to achieve these levels of apoptosis.

B

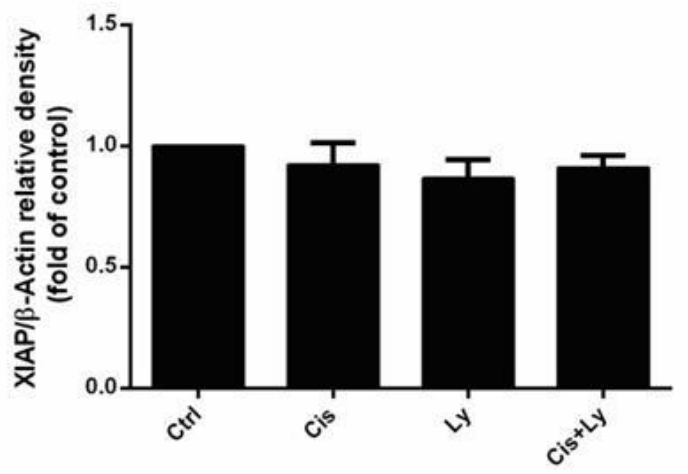

D

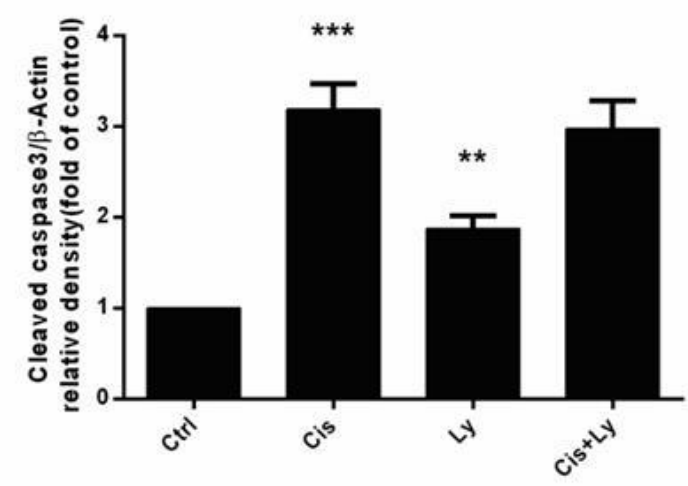

Fig. 3. The protein expression level of XIAP, caspase 3, and cleaved caspase 3 when they were treated with cisplatin, Ly alone, and their combination in the OVCAR3 cells and examined with western blotting assay. A) Western blotting image of XIAP, procaspase 3, cleaved caspase 3, and $\beta$-actin proteins determined via Western blot. Quantification of band densities, B), C), and D) protein expression fold of control of XIAP, pro-caspase 3, and cleaved caspase 3, respectively. The data represent the means \pm SEM of three separate experiments. $* \mathrm{p}<0.05, * * \mathrm{p}<0.01$ and $* * * \mathrm{p}<0.001$ versus Ctrl group.

\section{Akt activity}

To investigate the effect of cisplatin, Ly and cotreatment of them (cisplatin + Ly) on the PI3K/Akt signaling pathway due to involvement in cisplatin-based chemotherapy resistance, immunoblotting tests were performed to determine the protein expression level of PI3K, p-Ak, Akt, and Survivin in OVCAR3 cancer cells (Fig. 4). As indicated by the obtained results, the expression of $\mathrm{PI} 3 \mathrm{~K}$ remained unchanged based on treatment with all the mentioned groups (Fig. 4B). Regarding P-Akt protein content, treatment with cisplatin not only did not reduce the protein expression ratio but also surprisingly, the significant increase was detected in comparison to the control cell $(\mathrm{p}<$ $0.05)$. In contrast, Ly $(\mathrm{p}<0.001)$ decreased the PAkt protein level sharply, confirming the strong inhibition of AKT 
phosphorylation. A significant decrease in PAKT levels was detected in cisplatin + Ly versus cisplatin alone $(\mathrm{p}<0.001)$. However, the difference between Ly and cisplatin + Ly was insignificant compared with Ly alone

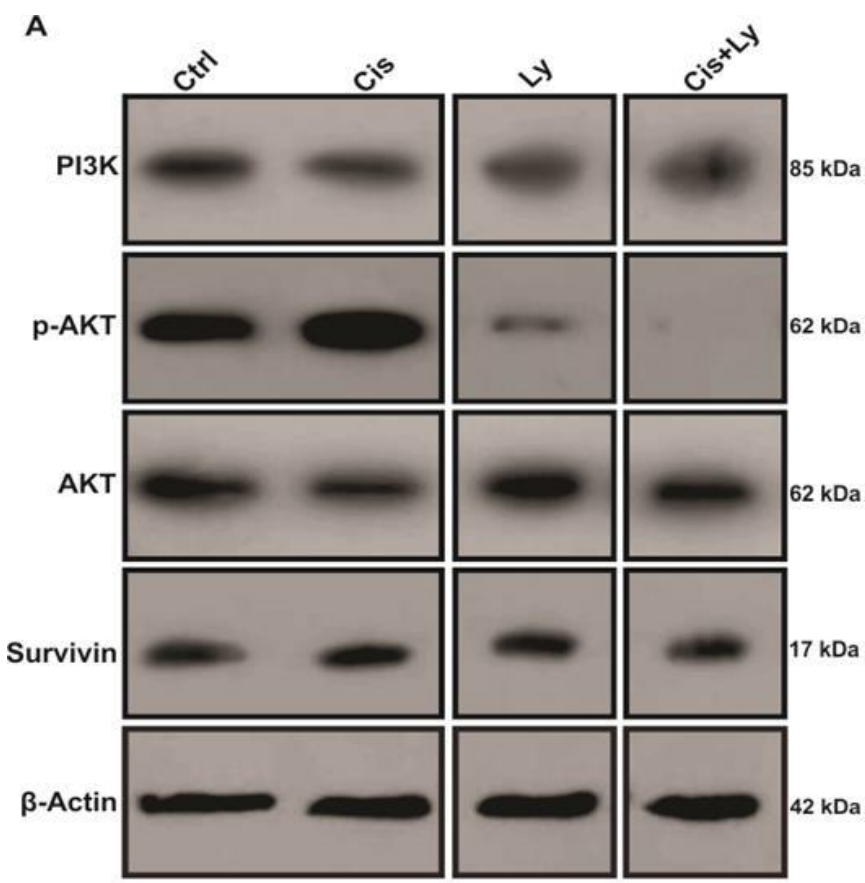

D

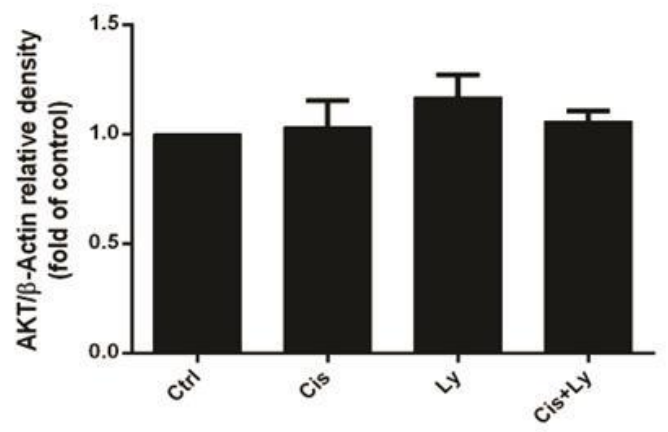

(Fig. 4C). Moreover, treatment with all studied groups neither did not alter the expression level of Akt nor survivin protein significantly in comparison with untreated OVCAR3 cells (Figs. 4D and 4E).

B

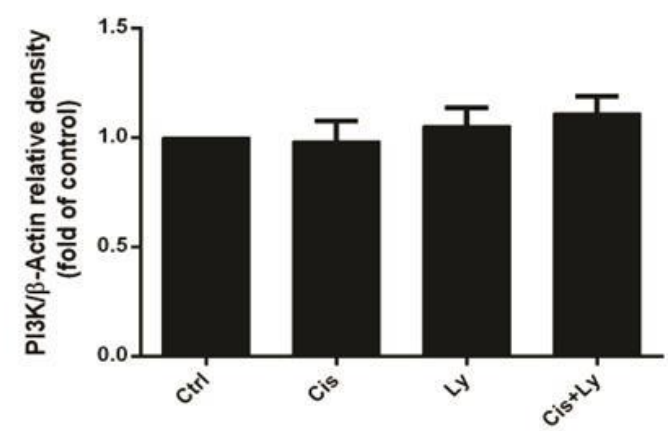

C

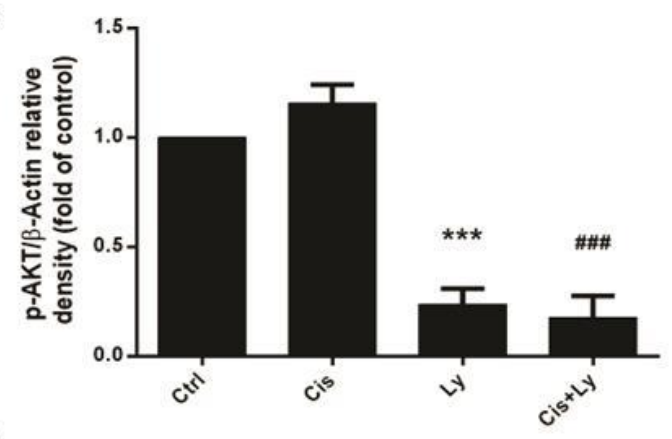

E

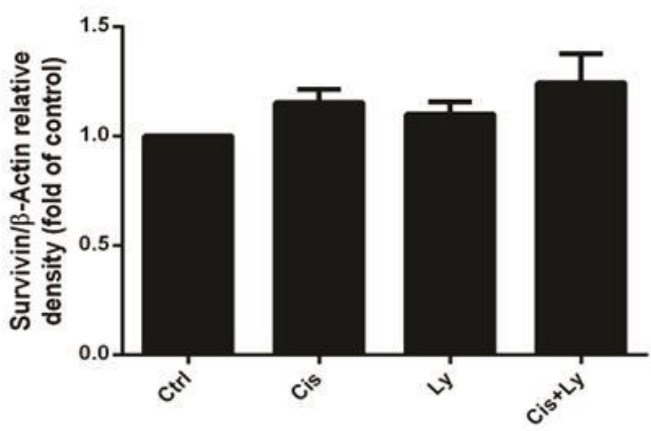

Fig. 4. The effect of cisplatin, Ly, and cisplatin plus Ly treatment on the expression level of proteins involved in the PI3K/Akt signaling pathway in the OVCAR3 cells. A) Immunoblotting image of all proteins, including PI3K, p-Akt, Akt, and Survivin. Besides, B), C), D), and E) the expression percentage of PI3K, p-Akt, Akt, and Survivin, respectively. The proteins were analyzed through western blotting assay, and the intensity of proteins band estimated based on the expression of $\beta$-actin as a control for protein loading. The experiments were repeated thrice as means $\pm \operatorname{SEM}(n=3)$ : ** $\mathrm{p}<0.001$ versus the control group. \#\# $\mathrm{p}<0.001$ versus Cis group. (Ctrl: control; Cis: cisplatin, Cis+Ly: Cisplatin + Ly).

\section{Discussion}

AKT/PI3K is a critical biological pathway involved in resistance to standard chemotherapeutic agents (19-21). Therefore, AKT/PI3K inactivation is of crucial importance in tumor prevention based on cytotoxic drugs and can be considered as one of the primary approaches for estimation of the clinical effectiveness of cancer cell sensitizing agents $(22,23)$. 
Cisplatin is a well-known anti-cancer agent named as cisplatinum or cisdiamminedichloroplatinum (II). Various types of human cancer, including head and neck, ovarian, bladder, lung, and testicular cancers, are being treated with cisplatin. The mechanism of action is related to the crosslinking ability of cisplatin with the purine bases on DNA molecules that led to intervention in DNA repair mechanism and, consequently, DNA damage and apoptosis induction in cancer cells. However, drug resistance, allergic reactions, decrease immunity to infections, gastrointestinal disorders, and hearing loss are serious problems limiting single therapy-based cisplatin. Therefore, co-administration of cisplatin, along with other types of anti-cancer drugs, can be considered as a novel therapy method to overcome drug resistance and reduce adverse side effects (24). LY294002 can reversibly inhibit 3 out of 4 isoforms of the catalytic subunit of class I in PI3K enzyme, $\mathrm{p} 110 \alpha, \mathrm{p} 110 \beta, \mathrm{p} 110 \gamma$, and $\mathrm{p} 110 \delta$. Ly acts on the ATP binding site of the catalytic subunit. The IC50s for $\mathrm{p} 110 \alpha, \beta$, and $\delta$ are $500 \mathrm{nM}, 973 \mathrm{nM}$, and $570 \mathrm{nM}(2,25,26) \mathrm{LY} 294002$ is also a potent autophagy inhibitor by blocking autophagosome formation (5).

In this study, we used combination therapy of Ly as a synthetic inhibitor of PI3K to block PI3K/Akt pathway along with cisplatin as a conventional chemotherapy agent to target multiple molecules in the OVCAR 3 cells.

In other words, the main aim of combination is down-regulation of cell survival pathway PI3K/Akt, up-regulation of apoptosis-related genes (caspases 3), and hyperactivation of oxidative stress.

Firstly, we compared the anti-proliferative effect of the combination of cisplatin and Ly with a single administration of cisplatin by MTT assay on the OVCAR3 cancer cell. The cell cytotoxicity results showed that administration of the $5.46 \mu \mathrm{M}$ cisplatin $+5 \mu \mathrm{M}$ Ly (IC50 in OVCAR3) inhibits the proliferation of ovcar3 cells as much as $13.18 \mu \mathrm{M}$ cisplatin alone. This result suggests that Akt inhibition by $\mathrm{Ly}$, in combination with cisplatin, could reduce the effective dose of cisplatin and therefore lower the side effects and drug resistance. However, the side effect of the Ly administration should also be investigated in comprehensive examines.

Phosphatidylinositol 3-kinase (PI3K) is an upstream protein of AKT, Phosphorylating Akt in response to various stimuli including protein phosphatase inhibitors, growth factor, stimulations, stress such as oxidation and Ras activation and extracellular matrix molecules $(27,28)$. PI3K performs a balance between cell survival and apoptosis, along with regulating various essential cellular responses $(29,30)$. After confirming of approximately complete inhibition of AKT phosphorylation in LY treated cells, Consistent with previous studies, we also found a remarkable decrease in AKT phosphorylation in cancer cells treated with Ly+cispaltin in proportion to cisplatin alone. This data is in line with the results of the MTT assay, which is mentioned above.

As aforementioned, the PI3K/Akt pathway contributes to developing ovarian cancer and induces chemoresistance to cisplatin and inhibits cisplatin-induced apoptosis of ovarian cancer cells (30-32). This study can be evidence of a slight increase in Akt protein level and phosphorylation of AKT in a single treatment of OVCAR3 cancer cells with cisplatin in our experiment. In a study, Lin et al. implied MK2206 as an inhibitor of the PI3K/Akt pathway in administrated with Taxol or cisplatin to assess the combined effect of

mentioned groups. The results depicted that inhibiting PI3K/Akt pathway enhanced the effects of cisplatin and Taxol, highlighting the critical roles of the Akt pathway in reversing chemoresistance in ovarian cancer cells (15).

ROS is a metabolic product of cellular aerobic metabolism that increases in its generation brings about mitochondrial damage, which consequently leads to cell death via apoptotic cascade reaction.

Previous studies have been shown that ROS production leads to apoptosis induction in ovarian cancer cells. Furthermore, ROS, as a crucial regulator of apoptosis, is contributed to a diverse array of pathological and physiological processes, including the 
mitochondria-mediated apoptosis pathway. The decrease in P-Akt protein expression in this study could increase the accumulation of intracellular ROS generation. Hence, our results prompt us to speculate that knockdown of PI3K/Akt signaling pathway via the combination treatment of cisplatin with Ly act as a leading factor of apoptosis induction via generation of ROS (17). In line with our obtained results YAMADA et al. depicted that down-regulation of PI3K/Akt signaling pathway via a combination of LY294002 as a synthetic inhibitor of PI3K and novel histone deacetylase (HDAC) inhibitor-induced ROS generation in RCC cancer cells (28).

Caspases are a group of intracellular cysteine proteases that are responsible for the activation of apoptosis signaling cascade. It has been reported that caspases are activated throughout apoptosis in various tumor cells. Cleaved caspase 3 as one of the main molecules is necessary for the onset of apoptosis irreversibly via both extrinsic and intrinsic pathways. Based on the intrinsic apoptotic pathway, cytochrome c release into cytosol and then binds to Apaf-1 and activates caspase- 9 , followed by caspase- 3 activation (33). We showed that treatment with Cisplatin, Ly, and co-administration of them reduced remarkably the content of pro-caspase 3 compared to control groups. In contrast, cleaved caspase 3 significantly increased in all treated cells in comparison to untreated cells, among which the effect of combination therapy was considerable compared to the cisplatintreated cells. This result shows that the hyper induction of apoptosis coincides with downregulation of P-Akt and hyperactivation of oxidative stress.

In line with our study, Lou et al. showed that treatment of cancer cells with Quercetin induces apoptosis via increasing the expression level of LC3 and ERK, cytoplasm p53, cleaved Caspase-3, and PARP and down-regulation protein expression of activated PI3K/AKT and Bcl-2 (34).

Previous studies have been shown that ROS production arises out of the down-regulation of Survivin and XIAP, which subsequently lead to apoptosis induction in ovarian cancer cells.
Besides, elevated X-linked IAP (XIAP) expression as a critical member of the inhibitor of apoptosis (IAP) family is of considerable significance in proliferation, cell invasion, and chemoresistance in ovarian cancer $(32,33)$. Overexpression of XIAP as a most potent caspases (including caspases-3, -7, and -9) inhibitory protein protects cancer cells bearing apoptosis induced with various anti-cancer agents $(22,35)$ in other hands, Survivin, as the other member of (IAP) family, display multiple functions, including regulation of apoptosis and mitosis. It is reported that the up-regulation of Survivin resulted in resistance of cancer cells toward conventional chemotherapeutic agents such as paclitaxel and cisplatin and, subsequently, prevention of tumor cells from apoptosis (28). Hence, suppression of survivin expression led to drug-induced apoptosis and also is one of the main indexes in opting novel anti-cancer drug. Based on such data we investigated the change in protein expression of XIAP and Survivin (34). Surprisingly we did not detect any significant decrease in the protein expression level of XIAP in three treated groups, including cisplatin, Ly, and LY+cisplatin, compared to control groups. Therefore, the activation of caspase 3 and decrease in cell proliferation and not change in $\mathrm{XIAP}$ and surviving expression promote us to assume that these molecules (XIAP and survivin) have been inactivated via an unknown way which deserves to investigate in future.

Overall, our obtained results displayed that co-treatment with cisplatin and Ly could sensitize the drug-resistant ovcar3 cancer cell line to cisplatin that is partially mediated by hyperactivation of oxidative stress.

Taken together, the results of this study depicted that the Ly could decrease the cisplatin -resistance in OVCAR3 cancer cells through the induction of apoptosis. It is noteworthy that the induction of apoptosis in combination therapy may be the result of an increase in ROS generation in OVCAR3 cancer cells.

\section{Acknowledgements}

We would like to give a special thanks to all individuals that have helped us in present study. 


\section{References}

1. Singh M, Chaudhry P, Fabi F, Asselin E. Cisplatin-induced caspase activation mediates PTEN cleavage in ovarian cancer cells: a potential mechanism of chemoresistance. BMC cancer. 2013; 13:233.

2. Bugide S, Gonugunta VK, Penugurti V, Malisetty VL, Vadlamudi RK, Manavathi B. HPIP promotes epithelial-mesenchymal transition and cisplatin resistance in ovarian cancer cells through PI3K/AKT pathway activation. Cell Oncol (Dordr). 2017;40(2):133-144.

3. Liu M, Bamodu OA, Huang WC, Zucha MA, Lin $\mathrm{YK}, \mathrm{Wu} \mathrm{ATH}$, et al. 4-Acetylantroquinonol B suppresses autophagic flux and improves cisplatin sensitivity in highly aggressive epithelial cancer through the PI3K/Akt/mTOR/p70S6K signaling pathway. Toxicol Appl Pharmacol. 2017;325:48-60.

4. Zhou B, Sun C, Li N, Shan W, Lu H, Guo L, et al. Cisplatin-induced CCL5 secretion from CAFs promotes cisplatin-resistance in ovarian cancer via regulation of the STAT3 and PI3K/Akt signaling pathways. Int J Oncol. 2016;48(5):2087-2097.

5. Li H, Xu H, Shen H, Li H. microRNA-106a modulates cisplatin sensitivity by targeting PDCD4 in human ovarian cancer cells. Oncol Lett. 2014;7(1):183-188.

6. Laatio L, Myllynen P, Serpi R, Rysä J, Ilves M, Lappi-Blanco E, et al. BMP-4 expression has prognostic significance in advanced serous ovarian carcinoma and is affected by cisplatin in OVCAR-3 cells. Tumour Biol. 2011;32(5):985-95.

7. Li B, Chen H, Wu N, Zhang WJ, Shang LX. Deregulation of miR-128 in ovarian cancer promotes cisplatin resistance. International Journal of Gynecologic Cancer. 2014;24(8):1381-1388.

8. Kim MG, Pak JH, Choi WH, Park JY, Nam $\mathrm{JH}$, Kim JH. The relationship between cisplatin resistance and histone deacetylase isoform overexpression in epithelial ovarian cancer cell lines. J Gynecol Oncol. 2012;23(3):182-189.

9. Ghaffari M, Dehghan G, Abedi-Gaballu F, Kashanian S, Baradaran B, Ezzati Nazhad Dolatabadi J, et al. Surface functionalized dendrimers as controlled-release delivery nanosystems for tumor targeting. Eur J Pharm Sci. 2018;122:311-330.

10. Fu X, Tian J, Zhang L, Chen Y, Hao Q. Involvement of microRNA-93, a new regulator of PTEN/Akt signaling pathway, in regulation of chemotherapeutic drug cisplatin chemosensitivity in ovarian cancer cells. FEBS Lett. 2012;586(9):1279-86.

11. Cai Y, Tan X, Liu J, Shen Y, Wu D, Ren M, et al. Inhibition of $\mathrm{PI} 3 \mathrm{~K} / \mathrm{Akt} / \mathrm{mTOR}$ signaling pathway enhances the sensitivity of the SKOV3/DDP ovarian cancer cell line to cisplatin in vitro. Chinese journal of cancer research. 2014; 26(5):564-72.

12. Gu J, Tang Y, Liu Y, Guo H, Wang Y, Cai L, et al. Murine double minute 2 siRNA and wildtype p53 gene therapy enhances sensitivity of the SKOV3/DDP ovarian cancer cell line to cisplatin chemotherapy in vitro and in vivo. Cancer Lett. 2014;343(2):200-9.

13. Liu J, Liu X, Ma W, Kou W, Li C, Zhao J. Anticancer activity of cucurbitacin-A in ovarian cancer cell line SKOV3 involves cell cycle arrest, apoptosis and inhibition of mTOR/PI3K/Akt signaling pathway. J BUON. 2018;23(1):124-128.

14. Choi HJ, Heo JH, Park JY, Jeong JY, Cho HJ, Park KS, et al. A novel PI3K/mTOR dual inhibitor, CMG002, overcomes the chemoresistance in ovarian cancer. Gynecologic oncol. 2019;153(1):135-148.

15. Lin YH, Chen BY, Lai WT, Wu SF, Guh JH, Cheng AL, et al. The Akt inhibitor MK-2206 enhances the cytotoxicity of paclitaxel (Taxol) and cisplatin in ovarian cancer cells. Naunyn Schmiedebergs Arch Pharmacol. 2015;388(1):19-31.

16. Qiu XM, Bai X, Jiang HF, He P, Wang JH. 20-(s)-ginsenoside $\operatorname{Rg} 3$ induces apoptotic cell death in human leukemic U937 and HL-60 cells through PI3K/Akt pathways. Anticancer Drugs. 2014;25(9):1072-80.

17. Kunnimalaiyaan $M$, Ndiaye $M$, Chen $H$. Apoptosis-mediated medullary thyroid cancer growth suppression by the $\mathrm{PI} 3 \mathrm{~K}$ inhibitor LY294002. Surgery. 2006;140(6):1009-14.

18. Farajdokht F, Mohaddes G, Karimi-Sales E, Kafshdooz T, Mahmoudi J, Aberoumandi SM. 
Inhibition of PTEN protects PC12 cells against oxygen-glucose deprivation induced cell death through mitoprotection. Brain Res. 2018;1692:100-109.

19. Zhang B, Wang X, Cai F, Chen W, Loesch U, Zhong XY. Antitumor properties of salinomycin on cisplatin-resistant human ovarian cancer cells in vitro and in vivo: involvement of $\mathrm{p} 38$ MAPK activation. Oncol Rep. 2013;29(4):1371-8.

20. Zhang Z, Xie Z, Sun G, Yang P, Li J, Yang $\mathrm{H}$, et al. Reversing drug resistance of cisplatin by hsp90 inhibitors in human ovarian cancer cells. Int J Clin Exp Med. 2015;8(5):6687-701.

21. Sapi E, Alvero AB, Chen W, O’Malley D, Hao X-Y, Dwipoyono B, et al. Resistance of ovarian carcinoma cells to docetaxel is XIAP dependent and reversible by phenoxodiol. Oncol Res. 2004;14(11-12): 567-78.

22. Paramasivam A, Sambantham S, Shabnam J, Raghunandhakumar S, Anandan B, Rajiv R, et al. Anti-cancer effects of thymoquinone in mouse neuroblastoma (Neuro-2a) cells through caspase3 activation with down-regulation of XIA. Toxicology Lett. 2012;213(2):151-9.

23. Hanifeh Ahagh M, Dehghan G, Mehdipour M, Teimuri-Mofrad R, Payami E, Sheibani N, et al. Synthesis, characterization, anti-proliferative properties and DNA binding of benzochromene derivatives: Increased $\mathrm{Bax} / \mathrm{Bcl}-2$ ratio and caspase-dependent apoptosis in colorectal cancer cell line. Bioorganic chemistry. 2019; 93:103329.

24. Dasari S, Tchounwou PB. Cisplatin in cancer therapy: molecular mechanisms of action. Eur J Pharmacol. 2014;740:364-78.

25. Fruman DA, Rommel C. PI3K $\delta$ inhibitors in cancer: rationale and serendipity merge in the clinic. Cancer Discov. 2011;1(7):562-72.

26. Pal I, Mandal M. PI3K and Akt as molecular targets for cancer therapy: current clinical outcomes. Acta Pharmacol Sin. 2012;33(12):1441-1458.

27. Ikezoe T, Nishioka C, Bandobashi K, Yang Y, Kuwayama Y, Adachi Y, et al. Longitudinal inhibition of $\mathrm{PI} 3 \mathrm{~K} / \mathrm{Akt} / \mathrm{mTOR}$ signaling by LY294002 and rapamycin induces growth arrest of adult T-cell leukemia cells. Leuk Res. 2007;31(5):673-82.
28. Yamada T, Horinaka $M$, Shinnoh $M$, Yoshioka T, Miki T, Sakai T. A novel HDAC inhibitor OBP-801 and a PI3K inhibitor LY294002 synergistically induce apoptosis via the suppression of survivin and XIAP in renal cell carcinoma. Int J Oncol. 2013;43(4):1080-6. 29. Rho SB, Kim BR, Kang S. A gene signaturebased approach identifies thioridazine as an inhibitor of phosphatidylinositol-3'-kinase (PI3K)/AKT pathway in ovarian cancer cells. Gynecol Oncol. 2011;120(1):121-7.

30. Osaki M, Kase S, Adachi K, Takeda A, Hashimoto K, Ito H. Inhibition of the PI3K-Akt signaling pathway enhances the sensitivity of Fas-mediated apoptosis in human gastric carcinoma cell line, MKN-45. J Cancer Res Clin Oncol. 2004;130(1):8-14.

31. Jiang H, Shang X, Wu H, Gautam SC, AlHolou S, Li C, et al. Resveratrol downregulates $\mathrm{PI} 3 \mathrm{~K} / \mathrm{Akt} / \mathrm{mTOR}$ signaling pathways in human U251 glioma cells. J Exp Ther Oncol. 2009;8(1):25-33.

32. Li Y, Zhang P, Qiu F, Chen L, Miao C, Li J, et al. Inactivation of PI3K/Akt signaling mediates proliferation inhibition and $\mathrm{G} 2 / \mathrm{M}$ phase arrest induced by andrographolide in human glioblastoma cells. Life sciences. 2012;90(25-26):962-7.

33. Mathiasen IS, Jäättelä M. Triggering caspase-independent cell death to combat cancer. Trends Mol Med. 2002;8(5):212-20.

34. Lou M, Zhang LN, Ji PG, Feng FQ, Liu JH, Yang C, et al. Quercetin nanoparticles induced autophagy and apoptosis through AKT/ERK/Caspase-3 signaling pathway in human neuroglioma cells: in vitro and in vivo. Biomed Pharmacother. 2016;84:1-9.

35. Xiong Z, Fu Z, Shi J, Jiang X, Wan H. HtrA1 down-regulation induces cisplatin resistance in colon cancer by increasing XIAP and activating PI3K/Akt pathway. Ann Clin Lab Sci. 2017;47(3):264-270. 